\title{
Conditional and unconditional co-occurrence restrictions in the recall of bigrams
}

\begin{abstract}
KIRK H. SMITH, 1 Bell Telephone Laboratories, Incorporated, Murray Hill, New Jersey
\end{abstract}

Previous investigations of the free recall of structured bigrams have concluded that $\mathrm{Ss}$ are unable to learn simple co-occurrence restrictions; however, only co-occurrence restrictions conditional upon the position in which each component letter occurs have been studied. Results of the present experiment suggest that Ss can and do make use of unconditional co-occurrence restrictions such as those found in a set of bigrams constructed from two classes of letters with the restriction that both letters appearing in a bigram must be drawn from the same class.

Recent studies (Segal \& Halwes, 1965, 1966; Smith, 1965, 1966) have shown that it is difficult to learn co-occurrence restrictions in the free recall of structured bigrams (letter pairs). In general, if the presented set contains bigrams having the forms $\mathrm{MN}$ and $P Q$, where $M, N, P$ and $Q$ represent nonoverlapping classes of three or four letters each, Ss will learn that $M$ and $P$ letters always come first and $\mathrm{N}$ and $\mathrm{Q}$ letters always come second, but they will fail to learn the co-occurrence restrictions, specifically, that $M$ letters can occur only if followed by $N$ letters, and $P$ letters only if followed by Q letters. Smith \& Gough (in press) have shown that this result is also obtained when the set of bigrams is designed to minimize positional regularities, which usually have a predominant influence on what is learned. They presented a set containing bigrams of the forms $\mathrm{AB}$ and $\mathrm{BA}$ and found that during recall $\mathrm{Ss}$ produced as many intrusions of the forms $A A$ and $B^{2}$ as intrusions of the forms $\mathrm{AB}$ and $\mathrm{BA}$. In fact, the only differences among intrusions (involving the letters which appeared in presented bigrams) were attributable to the fact that some intrusions were the result of reversing a presented bigram. While this reversal principle was readily learned, the restriction to bigrams of the forms $A B$ and $B A$ was not discernible in the pattern of intrusions Ss produced.

It is not clear why co-occurrence restrictions should be so difficult to learn, especially in view of the fact that similar restrictions occur throughout natural language. It should be noted that the co-occurrences in the Smith and Gough study are conditional upon position. An A letter can occur in first position only if a B letter appears in second position. But in the absence of unconditional positional regularities, which require that a class of letters appear in just one position, the $S$ may ignore all positional regularities, in particular those which are sensitive to context. The Smith and Gough bigram set provides neither unconditional positional regularities nor unconditional co-occurrence restrictions. A set of bigrams consisting of AA and BB combinations appears to provide the latter insofar as it requires that $S$ learn which letters may appear together without imposing any additional restrictions in terms of position. This previously unexploited design is investigated in the present study.

\section{METHOD}

Six lists of 32 bigrams were constructed from the 10 consonant letters, d, f, g, h, k, l, m, r, v, x, as follows: Three different orders of the letters were selected in a haphazard fashion, and each order was used to assign the letters to the rows and columns of a 10 by 10 matrix. For the three lists of Condition AABB, the 32 bigrams are represented by the solid cells of the matrix shown in Fig. 1a, where rows of the matrix represent bigrams with the same first letter and columns represent bigrams with the same second letter. The three lists of Condition ABBA were similarly constructed on the basis of the filled cells of Fig. $1 \mathrm{~b}$. It may be seen that the bigrams used in Condition $A A B B$ have the forms $A A$ and $B B$ and that those used in Condition $A B B A$ have the forms $A B$ and $B A$, when Class $A$ consists of the letters assigned to the first five rows and columns of the matrix and Class $B$ consists of the letters assigned to the second five rows and columns.

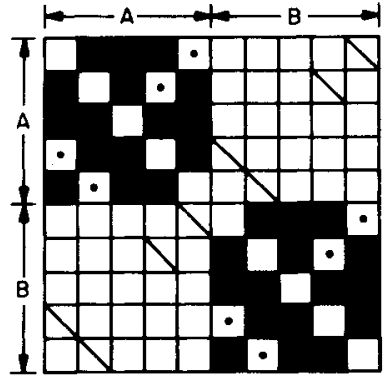

a

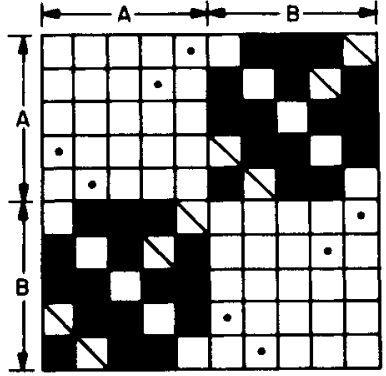

b
Fig. 1. Matrix representation of presented bigrams for Condition AABB (a) and Condition ABBA (b). Rows represent bigrams with the same first letter; columns, bigrams with the same second letter. Filled cells represent presented bigrams, and dots and diagonal lines indicate sets of intrusions given special analysis described in the text.

Each list was presented aurally by means of a tape recording to eight $S s$ in groups ranging in size from one to six. Following a single presentation of each of the 32 bigrams, Ss were instructed to write down as many of the bigrams as they could remember in any order on a sheet of paper numbered from 1 to 32 . There were four trials consisting of presentation followed by 3 min of written free recall; the order of presentation was randomly permuted on each trial.

The Ss were 48 students on the Madison, New Jersey, campus of Fairleigh-Dickenson University, who were paid for their time. RESULTS AND DISCUSSION

The mean number of presented bigrams correctly recalled per $S$ per trial was 14.97 for Condition AABB and 11.72 for Condition ABBA $(p<.01)$. The linear trend of the difference between the two conditions, showing an increase over trials, was also significant at the .01 level. There were no significant differences between the two conditions in the number of intrusions involving letters which appeared in presented bigrams. The mean number of such intrusions per $S$ per trial for Condition AABB was 5.21 and for Condition ABBA, 5.57.

If the enhanced recall shown in Condition AABB is due to Ss learning the co-occurrence restrictions implicit in the bigrams presented in this condition, then related trends would be expected in the production of intrusions of the forms $A A, B B, A B$, and $B A$. The production of $A A$ and $B B$ intrusions should exceed $A B$ and $B A$ intrusions by a greater amount for $S s$ in Condition $A A B B$ than $A B$ and $B A$ intrusions exceed $A A$ and $B B$ intrusions for $S s$ in Condition ABBA. This prediction is based upon an extension of earlier findings for verbal reconstructive memory (e.g., in the studies cited above). It has been shown that Ss will produce errors which extend certain structural regularities present in the bigrams to which they have been exposed if an opportunity for such intrusions is provided.

The structure of the presented bigrams was designed so that for each assignment of letters to the rows and columns of the 10 by 10 matrix, four combinations each of potential $A A$ and $B B$ intrusions (represented by cells containing dots in Fig. 1) and of potential $\mathbf{A B}$ and $\mathrm{BA}$ intrusions (represented by cells containing diagonal lines in Fig. 1) could be chosen for analysis. For a given letter assignment none of these combinations appeared among the presented bigrams; however, for Condition AABB, AA and BB combinations were reversals of presented bigrams and, similarly, for Condition $\mathrm{ABBA}$, the $\mathrm{AB}$ and $\mathrm{BA}$ combinations were reversals of presented bigrams.

Under Condition AABB, Ss produced a mean per trial of .72 $\mathrm{AA}$ and $\mathrm{BB}$ intrusions and $.29 \mathrm{AB}$ and $\mathrm{BA}$ intrusions from the selected subsets of four each described above. Corresponding 


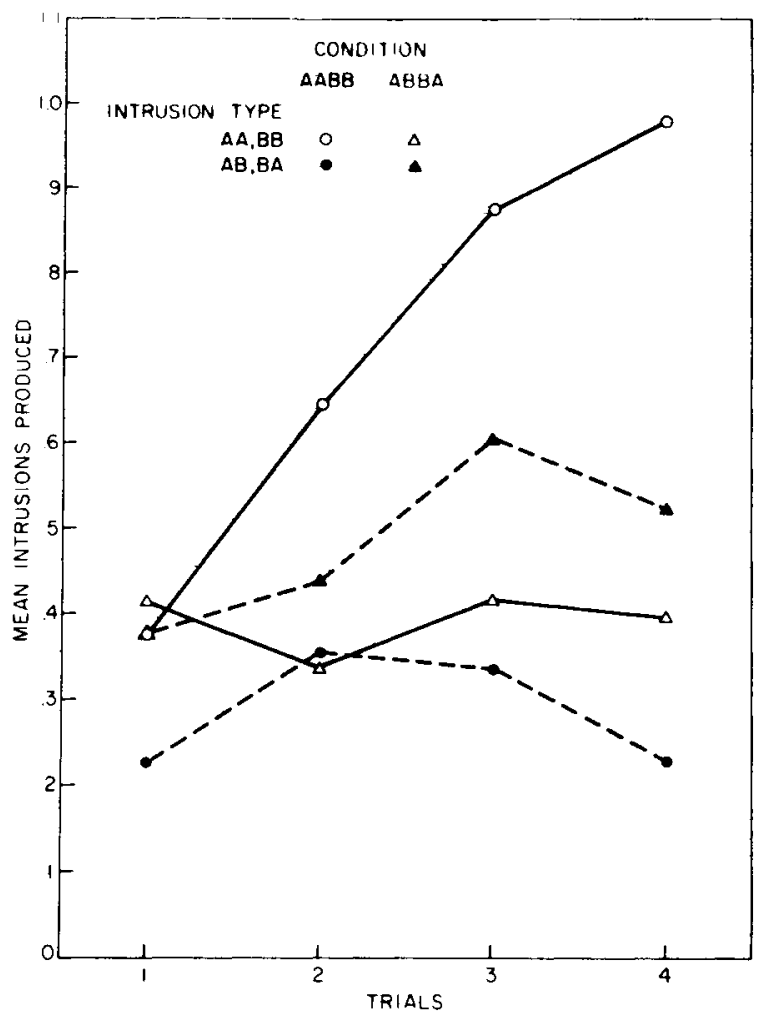

Fig. 2. Mean number of intrusions produced on four recall trials by $S s$ in two experimental conditions, AABB and ABBA. Each curve represents $24 \mathrm{Ss}$ and the average of two types of intrusions ( $A A$ and $B B$, or $A B$ and $B A$ ), where there were four potential intrusion combinations of each type.

intrusion rates for Condition ABBA were $.39 \mathrm{AA}$ and $\mathrm{BB}$ intrusions and $.48 \mathrm{AB}$ and $\mathrm{BA}$ intrusions. This constitutes a significant interaction $(p<.001)$ in the predicted direction. The magnitude of the interaction increased with repeated exposure of the materials. A significant linear trend of the interaction with trials (an orthogonal component of the Conditions by Intrusion Types by Trials interaction with $1 \mathrm{df}, \mathrm{p}<.001$ ) can be seen in the curves of Fig. 2. It is obvjous that the trend is primarily attributable to an increase in the number of $\mathrm{AA}$ and $\mathrm{BB}$ intrusions produced under Condition AABB. Moreover, the mean number of $\mathrm{AA}$ and $\mathrm{BB}$ intrusions for both conditions (.55) significantly exceeded $(p<.01)$ the overall mean number of $A B$ and $B A$ intrusions (.39). Given the direction of the differences tor the conditions separately. the latter contrast in effect compares the absolute magnitude of the differences under the two conditions.

Since the overall intrusion production did not differ between the two conditions, the intrusion data reported above cannot be explained by a differential overall propensity to produce intrusions. (More intrusions of all types were produced by Ss in Condition ABBA, although this difference did not approach significance.) The use of three different assignments of letters resulted in sets of bigrams differing both in the specific letter combinations presented for recall and in the letter combinations used to assess the intrusion rates among the four types of intrusions. The interaction of Conditions by Intrusion Types by Letter Assignments was not significant, nor was the four-way interaction involving Trials; therefore, the results cannot be readily explained in terms of either the specific letter combinations involved or such psychologically relevant properties of these combinations as frequency, associative strength, pronunciability, etc.

The results appear to confirm the expectation that Ss can discover and use the unconditional co-occurrence restrictions of Condition AABB whereas they are unresponsive to co-occurrence restrictions, such as those found in Condition $\mathrm{ABBA}$, that recuire concurrent sensitivity to position. The foregoing account of the differences between the two conditions is not intended to exclude the possibility that $S$ s noted other regularities and capitalized on them during recall. In particular, there is no reason to suppose that Ss did not perceive and utilize the reversal principle which applied to 24 of the 32 presented bigrams. However, the presence of this regularity was common to both conditions, and its utilization in the Ss' reconstructive memory processes should be regarded as complementary to the learning of co-occurrence restrictions. In a weak test of whether the Ss in Condition ABBA were utilizing the reversal principle, all bigram production scores were adjusted for differences in the total number of potential bigrams in each class and a comparison was made between $A B$ and $B A$ intrusions which did and did not have presented reversal counterparts. The former significantly exceded the latter $(p<.05)$, thus corroborating the earlier results of Smith and Gough.

\section{REFERENCES}

SEGAL, E. M., \& HALWES, T. G. Learning of letter pairs as a prototype of first language learning. Psychonomic Science, 1965, 3, 451-452.

SEGAL, E. M., \& HALWES. T. G. The influence of frequency of exposure on the learning of a phrase structural grammar. Psychonomic Science, 1966, 4, 157.158.

SMITH, K. H. Mediation and position learning in the recall of structured letter pairs. Psychonomic Science, 1965, 2, 293-294.

SMITH, K. H. Grammatical intrusions in the recall of structured letter pairs: Mediated transfer or position learning? Journal of Experimental Psychology, 1966, 72, 580-588.

SMITH, K. H., \& GOUGH, P. B. Transformation rules in the learning of miniature linguistic systems. Journal of Experimental Psychology, in press. NOTES

1. I would like to acknowledge a number of valuable discussions with Stuart Juuti concerning the problem to which this study is addressed. I am also indebted to Roseanne Speelman who collected the data for the experiment.

2. The notation is intended to imply that while both letters in the bigram are drawn from the same class, identical letters do not appear together. 